Supporting Information 


\section{Photophysical Diversity of Water Soluble Fluorescent Conjugated Polymers Induced by Surfactant Stabilizers for Rapid and Highly Selective Determination of 2,4,6-Trinitrotoluene Traces}

Naader Alizadeh*, Alireza Akbarinejad and Arash Ghoorchian

Department of Chemistry, Faculty of Basic Sciences, Tarbiat Modares University, P.O. Box

14115-175, Tehran, Iran

Corresponding author

*E-mail: alizaden@modares.ac.ir

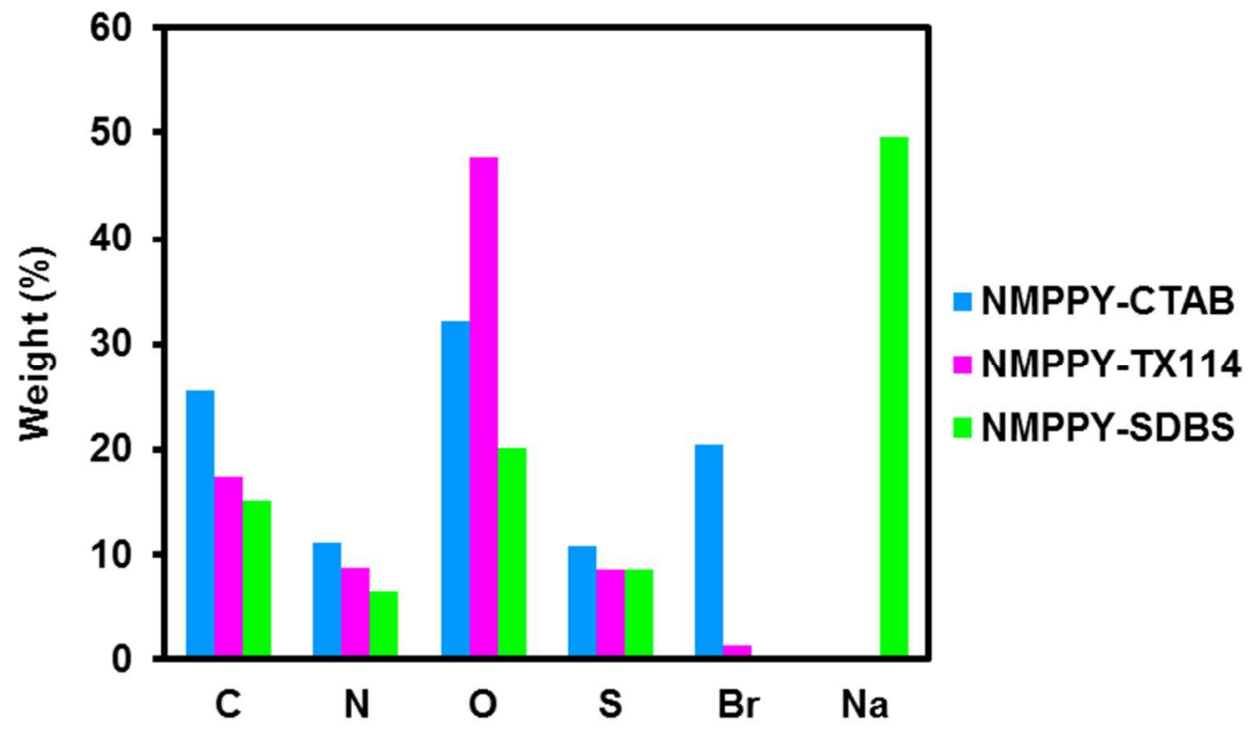

Fig. S1 Weight percentage of different elements in conjugated polymers measured by EDS analysis. 


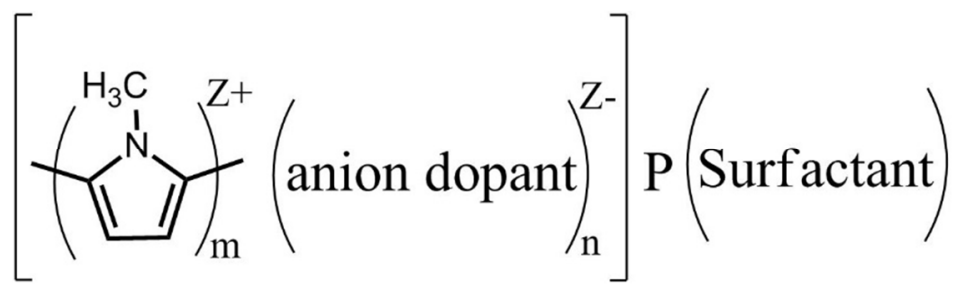

Fig. S2. Chemical structure of NMPPY-SDBS polymer.
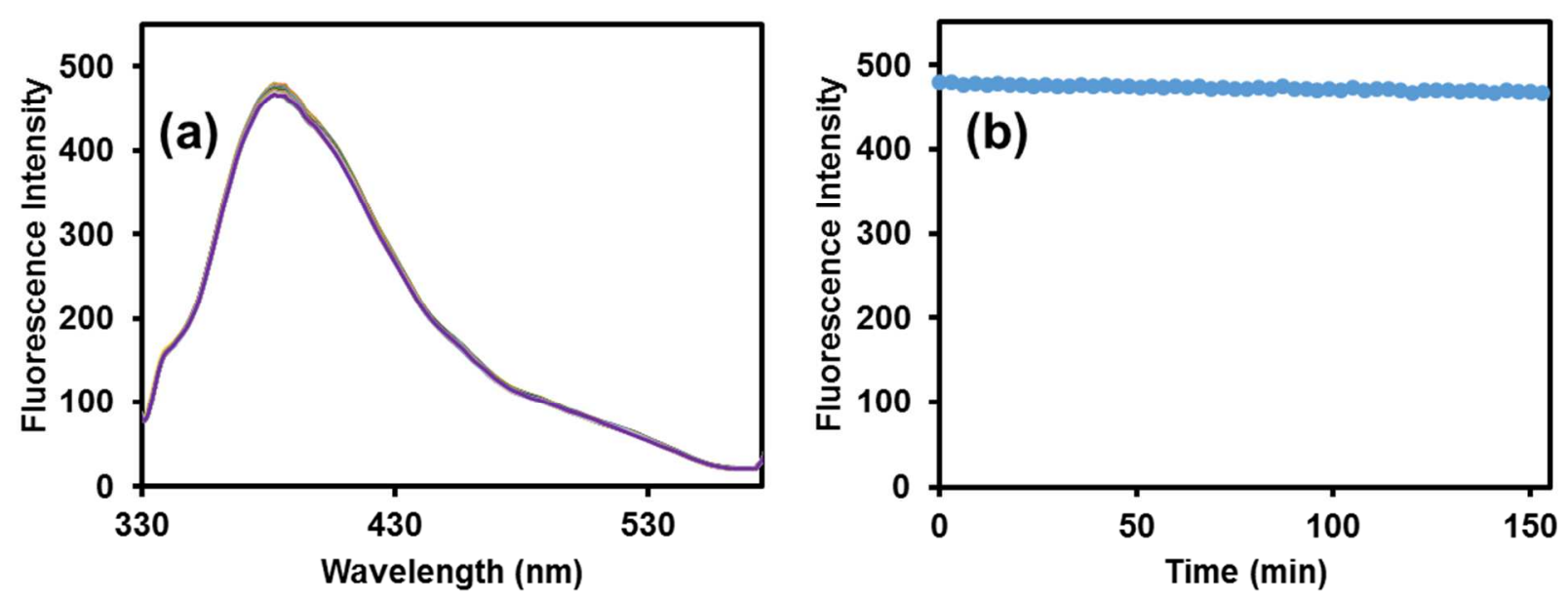

Fig. S3 (a) Fluorescence emission spectra of NMPPY-SDBS over a time period of $156 \mathrm{~min}$. (b) The changes in the fluorescence emission intensity of NMPPY-SDBS at $390 \mathrm{~nm}$ over the time period of $156 \mathrm{~min}$. 

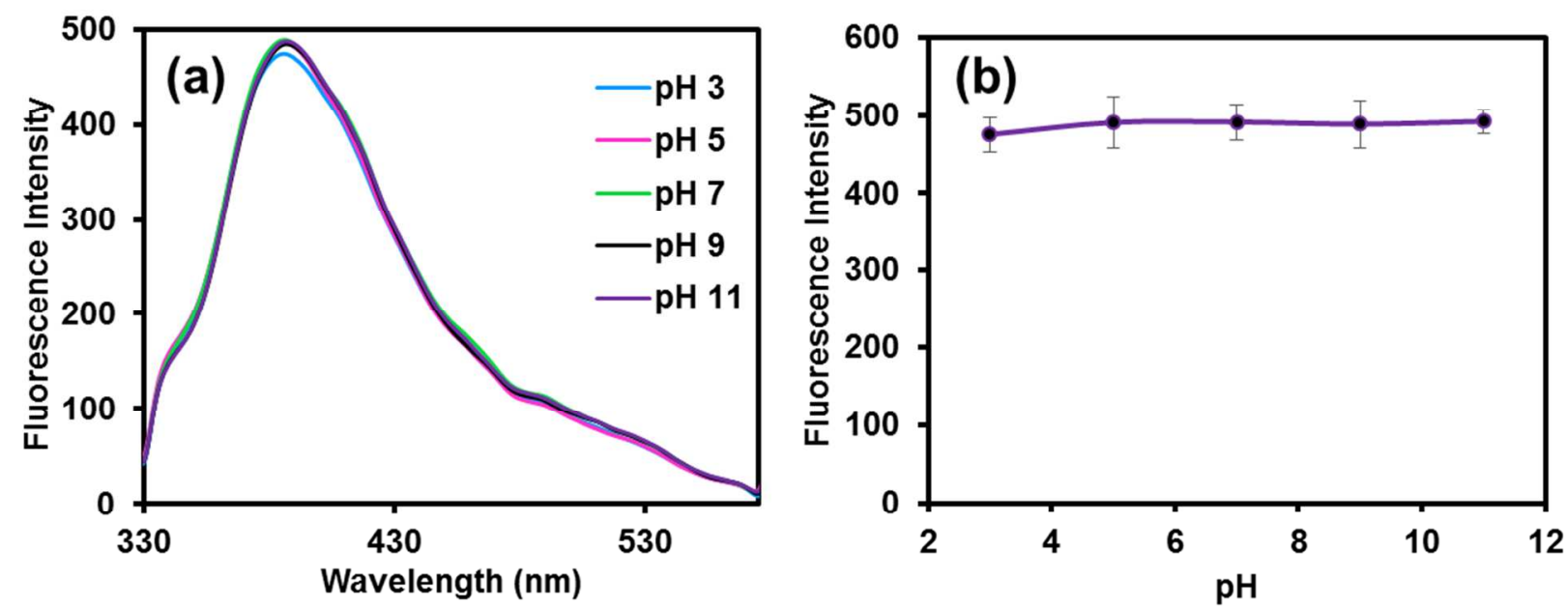

Fig. S4 (a) Fluorescence emission spectra of NMPPY-SDBS at pH values of 3, 5, 7, 9 and 11. (b) The changes in the fluorescence emission intensity of NMPPY-SDBS at $390 \mathrm{~nm}$ with varying the solution $\mathrm{pH}$.
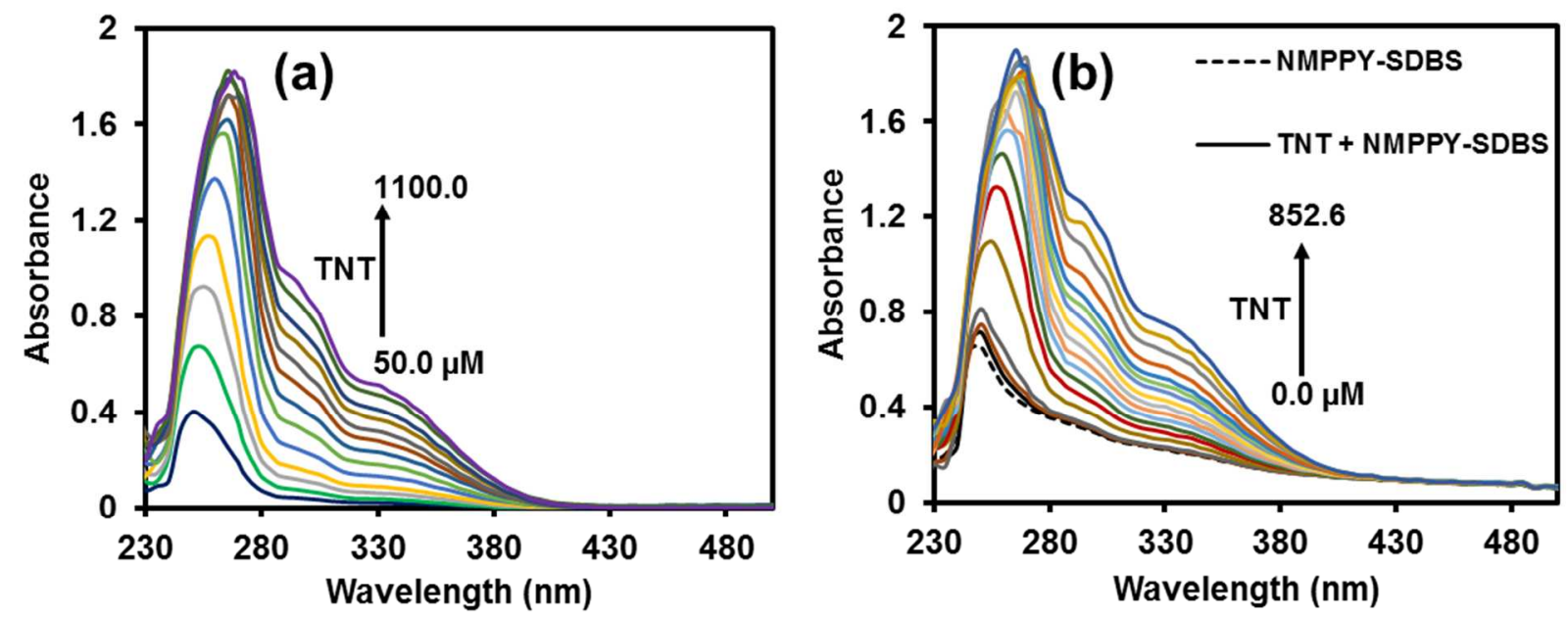

Fig. S5 UV-vis spectra of different concentrations of TNT in the absence (a) and presence (b) of NMPPY-SDBS in chloroform. 


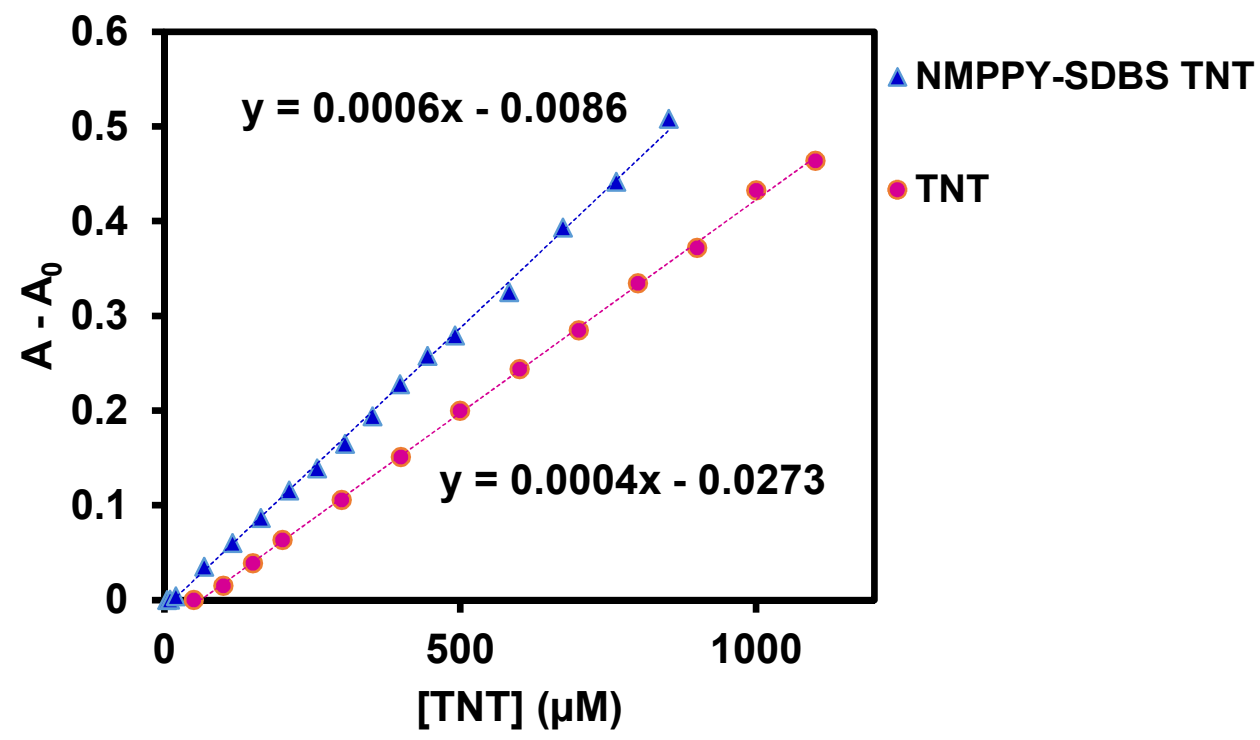

Fig. S6 The changes in absorbance at $335 \mathrm{~nm}$ upon addition of different concentrations of TNT in the presence and absence of NMPPY-SDBS in chloroform.

Table S1. Comparison of LOD of the proposed method and other techniques in determination of TNT.

\begin{tabular}{l|c|c|}
\multicolumn{1}{c}{ System } & $\begin{array}{c}\text { LOD } \mathbf{~ M , ~} \\
\text { (ppb) }\end{array}$ & $\begin{array}{c}\text { Reference } \\
\text { (Year) }\end{array}$ \\
\hline water-soluble pyrene derivatives & 801 & $\mathrm{~S}^{1}(2016)$ \\
electrospun pyrene-polyethersulfone nanofibers & 160 & $\mathrm{~S}^{2}(2015)$ \\
metal-organic frameworks & 460 & $\mathrm{~S}^{3}(2015)$ \\
phosphonate appended porphyrins & 5 & $\mathrm{~S}^{4}(2015)$ \\
phenylethynylene calix[4]arenes & 300 & $\mathrm{~S}^{5}(2015)$ \\
cross-linking electropolymerized films & 10000 & $\mathrm{~S}^{6}(2014)$ \\
molybdenum oxide quantum dots & 120 & $\mathrm{~S}^{7}(2016)$ \\
graphene quantum dots & 2200 & $\mathrm{~S}^{8}(2012)$ \\
tetraphenylethylene pillar[6]arenes & 100 & $\mathrm{~S}^{9}(2014)$ \\
water soluble conjugated polymer nanoparticles & 100 & This Work
\end{tabular}




\section{References:}

(S1) Kovalev, I. S.; Taniya, O. S.; Slovesnova, N. V.; Kim, G. A.; Santra, S.; Zyryanov, G. V.; Kopchuk, D. S.; Majee, A.; Charushin, V. N.; Chupakhin, O. N. Fluorescent Detection of 2,4-DNT and 2,4,6-TNT in Aqueous Media by Using Simple Water-Soluble Pyrene Derivatives Chem. Asian J. 2016, 11, 775-781.

(S2) Sun, X.; Liu, Y.; Shaw, G.; Carrier, A.; Dey, S.; Zhao, J.; Lei, Y. Fundamental Study of Electrospun Pyrene-Polyethersulfone Nanofibers Using Mixed Solvents for Sensitive and Selective Explosives Detection in Aqueous Solution ACS Appl. Mater. Interfaces 2015, 7, 13189-13197.

(S3) Yang, J.; Wang, Z.; Hu, K.; Li, Y.; Feng, J.; Shi, J.; Gu, J. Rapid and Specific Aqueous-Phase Detection of Nitroaromatic Explosives with Inherent Porphyrin Recognition Sites in MetalOrganic Frameworks ACS Appl. Mater. Interfaces 2015, 7, 11956-11964.

(S4) Venkatramaiah, N.; Pereira, C. F.; Mendes, R. F.; Paz, F. A. A.; Tomé, J. P. C. Phosphonate Appended Porphyrins as Versatile Chemosensors for Selective Detection of Trinitrotoluene Anal. Chem. 2015, 87, 4515-4522.

(S5) Boonkitpatarakul, K.; Yodta, Y.; Niamnont, N.; Sukwattanasinitt, M. Fluorescent Phenylethynylene Calix[4]arenes for Sensing TNT in Aqueous Media and Vapor Phase RSC Adv. 2015, 5, 33306-33311.

(S6) Nie, H.; Lv, Y.; Yao, L.; Pan, Y.; Zhao, Y.; Li, P.; Sun, G.; Ma, Y.; Zhang, M. Fluorescence Detection of Trace TNT by Novel Cross-Linking Electropolymerized Films Both in Vapor and Aqueous Medium J. Hazard. Mater. 2014, 264, 474-48o.

(S7) Xiao, S. J.; Zhao, X. J.; Hu, P. P.; Chu, Z. J.; Huang, C. Z.; Zhang, L. Highly Photoluminescent Molybdenum Oxide Quantum Dots: One-Pot Synthesis and Application in 2,4,6-Trinitrotoluene Determination ACS Appl. Mater. Interfaces 2016, 8, 8184-8191.

(S8) Fan, L.; Hu, Y.; Wang, X.; Zhang, L.; Li, F.; Han, D.; Li, Z.; Zhang, Q.; Wang, Z.; Niu, L. Fluorescence Resonance Energy Transfer Quenching at the Surface of Graphene Quantum Dots for Ultrasensitive Detection of TNT. Talanta 2012, 101, 192-197.

(S9) Wang, J.-H.; Feng, H.-T.; Zheng, Y.-S., Synthesis of Tetraphenylethylene Pillar[6]arenes and the Selective Fast Quenching of Their AIE Fluorescence by TNT Chem. Commun. 2014, 50, 1140711410. 